\title{
A Comparative Study on Fourth Order and Butcher's Fifth Order Runge-Kutta Methods with Third Order Initial Value Problem (IVP)
}

\author{
Md. Babul Hossain ${ }^{1, ~ *, ~ M d . ~ J a h a n g i r ~ H o s s a i n ~}{ }^{2}$, Md. Musa Miah ${ }^{1}$, Md. Shah Alam \\ ${ }^{1}$ Department of Mathematics, Mawlana Bhashani Science and Technology University, Tangail-1902, Bangladesh \\ ${ }^{2}$ Department of Basic Science, World University of Bangladesh, Dhaka, Bangladesh
}

Email address:

babulhossainh@yahoo.com (Md. B. Hossain)

*Corresponding author

\section{To cite this article:}

Md. Babul Hossain, Md. Jahangir Hossain, Md. Musa Miah, Md. Shah Alam. A Comparative Study on Fourth Order and Butcher's Fifth Order Runge-Kutta Methods with Third Order Initial Value Problem (IVP). Applied and Computational Mathematics.

Vol. 6, No. 6, 2017, pp. 243-253. doi: 10.11648/j.acm.20170606.12

Received: September 26, 2017; Accepted: October 8, 2017; Published: November 8, 2017

\begin{abstract}
In this paper, Butcher's fifth order Runge-Kutta (RK5) and fourth order Runge-Kutta (RK4) methods have been employed to solve the Initial Value Problems (IVP) involving third order Ordinary Differential Equations (ODE). These two proposed methods are quite proficient and practically well suited for solving engineering problems based on such problems. To obtain the accuracy of the numerical outcome for this study, we have compared the approximate results with the exact results and found a good agreement between the exact and approximate solutions. In addition, to achieve more accuracy in the solution, the step size needs to be very small. Moreover, the error terms have been analyzed for these two methods and also compared by an appropriate example.
\end{abstract}

Keywords: Differential Equation, Initial Value Problem, Error, Butcher's Method, Runge-Kutta Method

\section{Introduction}

Most of the branches in science, economics, management, engineering etc. most problems are formulated by differential equations. Most of the equations do not have analytical solutions, adequate to the development of numerical techniques. To solve these problems numerical approximations are convenient. For finding numerical approximations many methods are developed. Initial Value Problem (IVP) and Boundary Value Problem (BVP) are two parts of differential equations, depending on the conditions indicated at the end points of the domain. There are many methods that yield approximate solutions of initial value problems of Ordinary Differential Equations (ODE), such as Euler's method developed by Leonhard Euler in 1768, Runge-Kutta method developed around 1900 by Carl Runge and Martin Wilhelm Kutta respectively. Many authors try to spread these methods to get higher accurate solution of Initial Value Problems (IVP). In [1] the author presented fifth order improved Runge-Kutta method for solving ordinary differential equation, in [2] the author presented on fifth order Runge-Kutta methods, also in [3] the author presented a comparative study on numerical solutions of Initial Value Problems (IVP) for Ordinary Differential Equations (ODE) with Euler and Runge-Kutta methods. Also in [4] - [15] presented various numerical methods for finding approximations of Initial Value Problems (IVP) in Ordinary Differential Equations (ODE).

In this paper, we converted the coefficients of Butcher's RK5 table to the general fifth order Runge-Kutta method. Also we use fourth order Runge-Kutta (RK4) and fifth order Runge-Kutta (RK5) methods for solving third order initial value problems in ordinary differential equations. Approximate results are compared to the exact results and compared between these two methods.

\section{Numerical Methods}

In this section we represent Runge-Kutta fourth order (RK4) and Butcher's Runge-Kutta fifth order (RK5) methods for solving initial value problem (IVP) of ordinary 
differential equations (ODEs). Next we will apply these two methods for solving third order initial value problem.

\subsection{Runge-Kutta Method}

Runge-Kutta method is a technique for approximating the solution of ordinary differential equation. This technique was developed around 1900 by mathematicians Carl Runge and Wilhelm Kutta. Runge-Kutta method is popular because of its relative simplicity, sufficiency, accuracy and efficiency and used in most computer programs for a differential equation. This method is distinguished by their order in the sense that agrees with Taylor's series solution up to terms of $h^{r}$ where $r$ is the order of the method.

\subsection{Runge-Kutta Fourth Order Method}

Consider the following initial value problem:

$$
\frac{d y}{d x}=f(x, y)
$$

with initial condition $y\left(x_{0}\right)=y_{0}$.

Now the method is based on computing $y_{k+1}$ as follows:

$$
y_{k+1}=y_{k}+\frac{h}{6}\left(f_{1}+2 f_{2}+2 f_{3}+f_{4}\right)
$$

where

$$
\begin{gathered}
x_{k+1}=x_{k}+h \\
f_{1}=f\left(x_{k}, y_{k}\right) \\
f_{2}=f\left(x_{k}+\frac{h}{2}, y_{k}+\frac{h}{2} f_{1}\right) \\
f_{3}=f\left(x_{k}+\frac{h}{2}, y_{k}+\frac{h}{2} f_{2}\right) \\
f_{4}=f\left(x_{k}+h, y_{k}+h f_{3}\right)
\end{gathered}
$$

For $k=0,1,2, \ldots \ldots \ldots \ldots \ldots \ldots$

\subsection{Fourth Order Runge-Kutta Method for Solving Initial \\ Value Problem (IVP) for the System of Three Differential Equations}

Consider the following system of differential equations:

$$
\begin{aligned}
& \frac{d x}{d t}=P(t, x, y, z) \\
& \frac{d y}{d t}=Q(t, x, y, z) \\
& \frac{d z}{d t}=R(t, x, y, z)
\end{aligned}
$$

with initial conditions $x\left(t_{0}\right)=x_{0}, y\left(t_{0}\right)=y_{0}$

$$
z\left(t_{0}\right)=z_{0} \text {. }
$$

The fourth order Runge-Kutta method will become as:

$$
x_{i+1}=x_{i}+\frac{h\left(k_{1}+2 k_{2}+2 k_{3}+k_{4}\right)}{6}
$$

$$
\begin{aligned}
& y_{i+1}=y_{i}+\frac{h\left(l_{1}+2 l_{2}+2 l_{3}+l_{4}\right)}{6} \\
& z_{i+1}=z_{i}+\frac{h\left(m_{1}+2 m_{2}+2 m_{3}+m_{4}\right)}{6}
\end{aligned}
$$

Where

$$
\begin{gathered}
t_{i+1}=t_{i}+h \\
k_{1}=P\left(t_{i}, x_{i}, y_{i}, z_{i}\right) \\
l_{1}=Q\left(t_{i}, x_{i}, y_{i}, z_{i}\right) \\
m_{1}=R\left(t_{i}, x_{i}, y_{i}, z_{i}\right) \\
k_{2}=P\left(t_{i}+\frac{h}{2}, x_{i}+\frac{h}{2} k_{1}, y_{i}+\frac{h}{2} l_{1}, z_{i}+\frac{h}{2} m_{1}\right) \\
l_{2}=Q\left(t_{i}+\frac{h}{2}, x_{i}+\frac{h}{2} k_{1}, y_{i}+\frac{h}{2} l_{1}, z_{i}+\frac{h}{2} m_{1}\right) \\
m_{2}=R\left(t_{i}+\frac{h}{2}, x_{i}+\frac{h}{2} k_{1}, y_{i}+\frac{h}{2} l_{1}, z_{i}+\frac{h}{2} m_{1}\right) \\
k_{3}=P\left(t_{i}+\frac{h}{2}, x_{i}+\frac{h}{2} k_{2}, y_{i}+\frac{h}{2} l_{2}, z_{i}+\frac{h}{2} m_{2}\right) \\
l_{3}=Q\left(t_{i}+\frac{h}{2}, x_{i}+\frac{h}{2} k_{2}, y_{i}+\frac{h}{2} l_{2}, z_{i}+\frac{h}{2} m_{2}\right) \\
m_{3}=R\left(t_{i}+\frac{h}{2}, x_{i}+\frac{h}{2} k_{2}, y_{i}+\frac{h}{2} l_{2}, z_{i}+\frac{h}{2} m_{2}\right) \\
k_{4}=P\left(t_{i}+h, x_{i}+h k_{3}, y_{i}+h l_{3}, z_{i}+h m_{3}\right) \\
\left.m_{i}+h, x_{i}+h k_{3}, y_{i}+h l_{3}, z_{i}+h m_{3}\right) \\
\left.+h, x_{i}+h k_{3}, y_{i}+h l_{3}, z_{i}+h m_{3}\right)
\end{gathered}
$$

For $i=0,1,2$

\subsection{Butcher's Fifth Order Runge-Kutta Method}

Consider the following initial value problem:

$$
\frac{d y}{d x}=f(x, y)
$$

with initial condition $y\left(x_{0}\right)=y_{0}$.

Now the method is based on computing $y_{k+1}$ as follows:

$$
y_{k+1}=y_{k}+\frac{h}{90}\left(7 f_{1}+32 f_{3}+12 f_{4}+32 f_{5}+7 f_{6}\right)
$$

Where

$$
\begin{aligned}
& x_{k+1}=x_{k}+h \\
& f_{1}=f\left(x_{k}, y_{k}\right)
\end{aligned}
$$

$$
f_{2}=f\left(x_{k}+\frac{1}{4} h, y_{k}+\frac{1}{4} f_{1} h\right)
$$

$$
\begin{gathered}
f_{3}=f\left(x_{k}+\frac{1}{4} h, y_{k}+\frac{1}{8} f_{1} h+\frac{1}{8} f_{2} h\right) \\
f_{4}=f\left(x_{k}+\frac{1}{2} h, y_{k}-\frac{1}{2} f_{2} h+f_{3} h\right)
\end{gathered}
$$




$$
\begin{gathered}
f_{5}=f\left(x_{k}+\frac{3}{4} h, y_{k}+\frac{3}{16} f_{1} h+\frac{9}{16} f_{4} h\right) \\
f_{6}=f\left(x_{k}+h, y_{k}-\frac{3}{7} f_{1} h+\frac{2}{7} f_{2} h+\frac{12}{7} f_{3} h-\frac{12}{7} f_{4} h+\frac{8}{7} f_{5} h\right)
\end{gathered}
$$

For $k=0,1,2$

\subsection{Butcher's Fifth Order Runge-Kutta Method for Solving Initial Value Problem (IVP) for the System of Three Differential Equations}

Assume that the following system of differential equations:

$$
\begin{aligned}
\frac{d x}{d t} & =P(t, x, y, z) \\
\frac{d y}{d t} & =Q(t, x, y, z) \\
\frac{d z}{d t} & =R(t, x, y, z)
\end{aligned}
$$

with initial conditions $x\left(t_{0}\right)=x_{0}, y\left(t_{0}\right)=y_{0}$,

$$
z\left(t_{0}\right)=z_{0} .
$$

The Butcher's fifth order Runge-Kutta method will become as:

$$
\begin{gathered}
x_{i+1}=x_{i}+\frac{h}{90}\left(7 k_{1}+32 k_{3}+12 k_{4}+32 k_{5}+7 k_{6}\right) \\
y_{i+1}=y_{i}+\frac{h}{90}\left(7 l_{1}+32 l_{3}+12 l_{4}+32 l_{5}+7 l_{6}\right) \\
z_{i+1}=z_{i}+\frac{h}{90}\left(7 m_{1}+32 m_{3}+12 m_{4}+32 m_{5}+7 m_{6}\right)
\end{gathered}
$$

where

$$
\begin{gathered}
t_{i+1}=t_{i}+h \\
k_{1}=P\left(t_{i}, x_{i}, y_{i}, z_{i}\right) \\
l_{1}=Q\left(t_{i}, x_{i}, y_{i}, z_{i}\right) \\
m_{1}=R\left(t_{i}, x_{i}, y_{i}, z_{i}\right) \\
k_{2}=P\left(t_{i}+\frac{1}{4} h, x_{i}+\frac{1}{4} k_{1} h, y_{i}+\frac{1}{4} l_{1} h, z_{i}+\frac{1}{4} m_{1} h\right) \\
l_{2}=Q\left(t_{i}+\frac{1}{4} h, x_{i}+\frac{1}{4} k_{1} h, y_{i}+\frac{1}{4} l_{1} h, z_{i}+\frac{1}{4} m_{1} h\right) \\
m_{2}=R\left(t_{i}+\frac{1}{4} h, x_{i}+\frac{1}{4} k_{1} h, y_{i}+\frac{1}{4} l_{1} h, z_{i}+\frac{1}{4} m_{1} h\right) \\
k_{3}=P\left(t_{i}+\frac{1}{4} h, x_{i}+\frac{1}{8} k_{1} h+\frac{1}{8} k_{2} h, y_{i}+\frac{1}{8} l_{1} h+\frac{1}{8} l_{2} h, z_{i}\right. \\
\left.+\frac{1}{8} m_{1} h+\frac{1}{8} m_{2} h\right) \\
l_{3}=Q\left(t_{i}+\frac{1}{4} h, x_{i}+\frac{1}{8} k_{1} h+\frac{1}{8} k_{2} h, y_{i}+\frac{1}{8} l_{1} h+\frac{1}{8} l_{2} h, z_{i}\right. \\
\left.+\frac{1}{8} m_{1} h+\frac{1}{8} m_{2} h\right)
\end{gathered}
$$

$$
\begin{aligned}
& m_{3}=R\left(t_{i}+\frac{1}{4} h, x_{i}+\frac{1}{8} k_{1} h+\frac{1}{8} k_{2} h, y_{i}+\frac{1}{8} l_{1} h+\frac{1}{8} l_{2} h, z_{i}\right. \\
& \left.+\frac{1}{8} m_{1} h+\frac{1}{8} m_{2} h\right) \\
& k_{4}=P\left(t_{i}+\frac{1}{2} h, x_{i}-\frac{1}{2} k_{2} h+k_{3} h, y_{i}-\frac{1}{2} l_{2} h+l_{3} h, z_{i}\right. \\
& \left.-\frac{1}{2} m_{2} h+m_{3} h\right) \\
& l_{4}=Q\left(t_{i}+\frac{1}{2} h, x_{i}-\frac{1}{2} k_{2} h+k_{3} h, y_{i}-\frac{1}{2} l_{2} h+l_{3} h, z_{i}\right. \\
& \left.-\frac{1}{2} m_{2} h+m_{3} h\right) \\
& m_{4}=R\left(t_{i}+\frac{1}{2} h, x_{i}-\frac{1}{2} k_{2} h+k_{3} h, y_{i}-\frac{1}{2} l_{2} h+l_{3} h, z_{i}\right. \\
& \left.-\frac{1}{2} m_{2} h+m_{3} h\right) \\
& k_{5}=P\left(t_{i}+\frac{3}{4} h, x_{i}+\frac{3}{16} k_{1} h+\frac{9}{16} k_{4} h, y_{i}+\frac{3}{16} l_{1} h\right. \\
& \left.+\frac{9}{16} l_{4} h, z_{i}+\frac{3}{16} m_{1} h+\frac{9}{16} m_{4} h\right) \\
& l_{5}=Q\left(t_{i}+\frac{3}{4} h, x_{i}+\frac{3}{16} k_{1} h+\frac{9}{16} k_{4} h, y_{i}+\frac{3}{16} l_{1} h\right. \\
& \left.+\frac{9}{16} l_{4} h, z_{i}+\frac{3}{16} m_{1} h+\frac{9}{16} m_{4} h\right) \\
& m_{5}=R\left(t_{i}+\frac{3}{4} h, x_{i}+\frac{3}{16} k_{1} h+\frac{9}{16} k_{4} h, y_{i}+\frac{3}{16} l_{1} h\right. \\
& \left.+\frac{9}{16} l_{4} h, z_{i}+\frac{3}{16} m_{1} h+\frac{9}{16} m_{4} h\right) \\
& k_{6}=P\left(t_{i}+h, x_{i}-\frac{3}{7} k_{1} h+\frac{2}{7} k_{2} h+\frac{12}{7} k_{3} h-\frac{12}{7} k_{4} h+\frac{8}{7} k_{5} h, y_{i}\right. \\
& -\frac{3}{7} l_{1} h+\frac{2}{7} l_{2} h+\frac{12}{7} l_{3} h-\frac{12}{7} l_{4} h+\frac{8}{7} l_{5} h \text {, } \\
& \left.z_{i}-\frac{3}{7} m_{1} h+\frac{2}{7} m_{2} h+\frac{12}{7} m_{3} h-\frac{12}{7} m_{4} h+\frac{8}{7} m_{5} h\right) \\
& l_{6}=Q\left(t_{i}+h, x_{i}-\frac{3}{7} k_{1} h+\frac{2}{7} k_{2} h+\frac{12}{7} k_{3} h-\frac{12}{7} k_{4} h+\frac{8}{7} k_{5} h, y_{i}\right. \\
& -\frac{3}{7} l_{1} h+\frac{2}{7} l_{2} h+\frac{12}{7} l_{3} h-\frac{12}{7} l_{4} h+\frac{8}{7} l_{5} h \text {, } \\
& \left.z_{i}-\frac{3}{7} m_{1} h+\frac{2}{7} m_{2} h+\frac{12}{7} m_{3} h-\frac{12}{7} m_{4} h+\frac{8}{7} m_{5} h\right) \\
& m_{6}=R\left(t_{i}+h, x_{i}-\frac{3}{7} k_{1} h+\frac{2}{7} k_{2} h+\frac{12}{7} k_{3} h-\frac{12}{7} k_{4} h+\frac{8}{7} k_{5} h, y_{i}\right. \\
& -\frac{3}{7} l_{1} h+\frac{2}{7} l_{2} h+\frac{12}{7} l_{3} h-\frac{12}{7} l_{4} h+\frac{8}{7} l_{5} h \\
& \left.z_{i}-\frac{3}{7} m_{1} h+\frac{2}{7} m_{2} h+\frac{12}{7} m_{3} h-\frac{12}{7} m_{4} h+\frac{8}{7} m_{5} h\right)
\end{aligned}
$$

For $i=0,1,2,3$,

\section{Error Analysis}

To finding the numerical solution of ordinary differential equations, two types of errors occurs. Round-off errors and Truncation errors. Rounding errors originate from the fact that computer can only represent numbers using a fixed and 
limited number of significant figures. Thus, such numbers cannot be represented exactly in computer memory. The discrepancy introduced by this limitation is called Round-off error. Truncation error in numerical analysis arises when approximations are used to estimate some quantity. The accuracy of the solution will depend on how small we take the step size $h$. A numerical method is said to be convergent if the numerical solution approaches the exact solution as the step size $h$ goes to 0 . In this paper, we consider a third order initial value problem to verify accuracy of the proposed method. Then using this method we find numerical approximations for desired initial value problems. The approximate solution is evaluated by using MATLAB software for two proposed numerical methods at different step sizes. The convergence of initial value problem is calculated by $e_{n}=\left|z\left(t_{n}\right)-z_{n}\right|<\delta$ where $z\left(t_{n}\right)$ denotes the approximate solution and $z_{n}$ denotes the exact solution and $\delta$ depends on the problem which varies from $10^{-1}$. The errors for these two formulas are defined by errors $=\left|z\left(t_{n}\right)-z_{n}\right|$.

\section{Applications of These Numerical Methods on Illustrative Example}

In this section we consider a third order initial value problem as numerical example to compare between these two methods. All the computations are performed by MATLAB software. Numerical results and errors are computed and the outcomes are represented by graphically.

Example:

Consider the initial value problem $\frac{d^{3} z}{d t^{3}}-5 \frac{d^{2} z}{d t^{2}}+8 \frac{d z}{d t}-$ $4 z=0$ with initial conditions $z(0)=1, z^{\prime}(0)=2, z^{\prime \prime}(0)=$ -1 on the interval $0 \leq t \leq 3$. The exact solution of the given problem is $z(t)=-5 e^{t}+(6-5 t) e^{2 t}$.

Now this third order initial value problem can be written in the form of system of first order differential equations by putting $y=\frac{d z}{d t}$ and $x=\frac{d y}{d t}=\frac{d^{2} z}{d t^{2}}$ as

$$
\begin{gathered}
\frac{d z}{d t}=y \\
\frac{d y}{d t}=x \\
\frac{d x}{d t}=5 x-8 y+4 z
\end{gathered}
$$

with initial conditions $z(0)=1, y(0)=2$ and $x(0)=-1$.

The approximate results, exact results and maximum errors for step sizes $0.1,0.05,0.025$ and 0.0125 are shown in Table

\begin{tabular}{|c|c|c|c|c|c|}
\hline \multirow{2}{*}{$t_{n}$} & \multicolumn{2}{|l|}{ RK4 Method h=0.1 } & \multicolumn{2}{|l|}{ RK5 Method h=0.1 } & \multirow{2}{*}{$\begin{array}{l}\text { Exact value } \\
z_{n}\end{array}$} \\
\hline & $z\left(t_{n}\right)$ & errors & $z\left(t_{n}\right)$ & errors & \\
\hline 0.00 & $1.000000000 \mathrm{E}+00$ & $0.0000 \mathrm{E}+00$ & $1.000000000 \mathrm{E}+00$ & $0.0000 \mathrm{E}+00$ & $1.000000000 \mathrm{E}+00$ \\
\hline 0.10 & $1.191879167 \mathrm{E}+00$ & $1.8587 \mathrm{E}-05$ & $1.191860509 \mathrm{E}+00$ & 7.0649E-08 & $1.191860580 \mathrm{E}+00$ \\
\hline 0.20 & $1.352158372 \mathrm{E}+00$ & $4.8675 \mathrm{E}-05$ & $1.352109515 \mathrm{E}+00$ & $1.8279 \mathrm{E}-07$ & $1.352109697 \mathrm{E}+00$ \\
\hline 0.30 & $1.450335750 \mathrm{E}+00$ & $9.5186 \mathrm{E}-05$ & $1.450240210 \mathrm{E}+00$ & $3.5363 \mathrm{E}-07$ & $1.450240564 \mathrm{E}+00$ \\
\hline 0.40 & $1.443205050 \mathrm{E}+00$ & $1.6482 \mathrm{E}-04$ & $1.443039619 \mathrm{E}+00$ & $6.0647 \mathrm{E}-07$ & $1.443040226 \mathrm{E}+00$ \\
\hline 0.50 & $1.270646705 \mathrm{E}+00$ & $2.6666 \mathrm{E}-04$ & $1.270379073 \mathrm{E}+00$ & $9.7266 \mathrm{E}-07$ & $1.270380046 \mathrm{E}+00$ \\
\hline 0.60 & $8.501696545 \mathrm{E}-01$ & 4.1289E-04 & $8.497552721 \mathrm{E}-01$ & $1.4942 \mathrm{E}-06$ & $8.497567663 \mathrm{E}-01$ \\
\hline 0.70 & 6.985621392E-02 & $6.1983 \mathrm{E}-04$ & $6.923415278 \mathrm{E}-02$ & $2.2270 \mathrm{E}-06$ & $6.923637976 \mathrm{E}-02$ \\
\hline 0.80 & $-1.220730555 \mathrm{E}+00$ & $9.0924 \mathrm{E}-04$ & $-1.221643039 \mathrm{E}+00$ & $3.2453 \mathrm{E}-06$ & $-1.221639794 \mathrm{E}+00$ \\
\hline 0.90 & $-3.222234415 \mathrm{E}+00$ & $1.3099 \mathrm{E}-03$ & $-3.223549006 \mathrm{E}+00$ & $4.6473 \mathrm{E}-06$ & $-3.223544359 \mathrm{E}+00$ \\
\hline 1.00 & $-6.200492971 E+00$ & $1.8601 \mathrm{E}-03$ & $-6.202359606 \mathrm{E}+00$ & $6.5622 \mathrm{E}-06$ & $-6.202353043 \mathrm{E}+00$ \\
\hline 1.10 & $-1.050571353 \mathrm{E}+01$ & $2.6098 \mathrm{E}-03$ & $-1.050833253 \mathrm{E}+01$ & $9.1599 \mathrm{E}-06$ & $-1.050832337 \mathrm{E}+01$ \\
\hline 1.20 & $-1.659695942 \mathrm{E}+01$ & $3.6252 \mathrm{E}-03$ & $-1.660059728 \mathrm{E}+01$ & $1.2663 \mathrm{E}-05$ & $-1.660058461 \mathrm{E}+01$ \\
\hline 1.30 & $-2.507335987 \mathrm{E}+01$ & 4.9925E-03 & $-2.507836972 \mathrm{E}+01$ & $1.7361 \mathrm{E}-05$ & $-2.507835236 \mathrm{E}+01$ \\
\hline 1.40 & $-3.671382206 \mathrm{E}+01$ & $6.8245 \mathrm{E}-03$ & $-3.672067024 \mathrm{E}+01$ & $2.3634 \mathrm{E}-05$ & $-3.672064661 \mathrm{E}+01$ \\
\hline 1.50 & $-5.252748238 \mathrm{E}+01$ & $9.2684 \mathrm{E}-03$ & $-5.253678271 \mathrm{E}+01$ & 3.1972E-05 & $-5.253675074 \mathrm{E}+01$ \\
\hline 1.60 & $-7.381770747 \mathrm{E}+01$ & $1.2515 \mathrm{E}-02$ & $-7.383026553 \mathrm{E}+01$ & $4.3015 \mathrm{E}-05$ & $-7.383022252 \mathrm{E}+01$ \\
\hline 1.70 & $-1.022631744 \mathrm{E}+02$ & $1.6813 \mathrm{E}-02$ & $-1.022800447 \mathrm{E}+02$ & $5.7590 \mathrm{E}-05$ & $-1.022799871 \mathrm{E}+02$ \\
\hline 1.80 & $-1.400204583 \mathrm{E}+02$ & $2.2482 \mathrm{E}-02$ & $-1.400430174 \mathrm{E}+02$ & 7.6764E-05 & $-1.400429407 \mathrm{E}+02$ \\
\hline 1.90 & $-1.898536787 \mathrm{E}+02$ & 2.9939E-02 & $-1.898837199 \mathrm{E}+02$ & $1.0192 \mathrm{E}-04$ & $-1.898836179 \mathrm{E}+02$ \\
\hline 2.00 & $-2.552981612 \mathrm{E}+02$ & 3.9719E-02 & $-2.553380155 \mathrm{E}+02$ & $1.3483 \mathrm{E}-04$ & $-2.553378806 \mathrm{E}+02$ \\
\hline 2.10 & $-3.408668260 \mathrm{E}+02$ & $5.2513 \mathrm{E}-02$ & $-3.409195170 \mathrm{E}+02$ & $1.7777 \mathrm{E}-04$ & $-3.409193392 \mathrm{E}+02$ \\
\hline 2.20 & $-4.523102017 \mathrm{E}+02$ & $6.9209 \mathrm{E}-02$ & $-4.523796445 \mathrm{E}+02$ & $2.3370 \mathrm{E}-04$ & $-4.523794108 \mathrm{E}+02$ \\
\hline 2.30 & $-5.969436997 \mathrm{E}+02$ & $9.0949 \mathrm{E}-02$ & $-5.970349547 \mathrm{E}+02$ & $3.0637 \mathrm{E}-04$ & $-5.970346483 \mathrm{E}+02$ \\
\hline 2.40 & $-7.840591906 \mathrm{E}+02$ & $1.1920 \mathrm{E}-01$ & $-7.841787876 \mathrm{E}+02$ & $4.0060 \mathrm{E}-04$ & $-7.841783870 \mathrm{E}+02$ \\
\hline 2.50 & $-1.025442174 \mathrm{E}+03$ & $1.5583 \mathrm{E}-01$ & $-1.025598527 \mathrm{E}+03$ & $5.2258 \mathrm{E}-04$ & $-1.025598004 \mathrm{E}+03$ \\
\hline 2.60 & $-1.336021133 \mathrm{E}+03$ & $2.0325 \mathrm{E}-01$ & $-1.336225064 \mathrm{E}+03$ & $6.8020 \mathrm{E}-04$ & $-1.336224383 \mathrm{E}+03$ \\
\hline 2.70 & $-1.734682248 E+03$ & 2.6453E-01 & $-1.734947664 \mathrm{E}+03$ & 8.8354E-04 & $-1.734946780 \mathrm{E}+03$ \\
\hline 2.80 & $-2.245290896 \mathrm{E}+03$ & $3.4360 \mathrm{E}-01$ & $-2.245635639 \mathrm{E}+03$ & $1.1455 \mathrm{E}-03$ & $-2.245634493 \mathrm{E}+03$ \\
\hline 2.90 & $-2.897971535 \mathrm{E}+03$ & $4.4545 \mathrm{E}-01$ & $-2.898418468 \mathrm{E}+03$ & $1.4824 \mathrm{E}-03$ & $-2.898416986 \mathrm{E}+03$ \\
\hline 3.00 & $-3.730710350 \mathrm{E}+03$ & $5.7648 \mathrm{E}-01$ & $-3.731288741 \mathrm{E}+03$ & $1.9151 \mathrm{E}-03$ & $-3.731286826 \mathrm{E}+03$ \\
\hline
\end{tabular}
1-4 and the graphs of the numerical solutions.

Table 1. Numerical approximations and maximum errors for step size $h=0.1$. 
Table 2. Numerical approximations and maximum errors for step size $h=0.05$.

\begin{tabular}{|c|c|c|c|c|c|}
\hline \multirow{2}{*}{$t_{n}$} & \multicolumn{2}{|l|}{ RK4 Method h=0.05 } & \multicolumn{2}{|l|}{ RK5 Method $\mathrm{h}=0.05$} & \multirow{2}{*}{$\begin{array}{l}\text { Exact value } \\
z_{n}\end{array}$} \\
\hline & $z\left(t_{n}\right)$ & errors & $z\left(t_{n}\right)$ & errors & \\
\hline 0.00 & $1.000000000 \mathrm{E}+00$ & $0.0000 \mathrm{E}+00$ & $1.000000000 \mathrm{E}+00$ & $0.0000 \mathrm{E}+00$ & $1.000000000 \mathrm{E}+00$ \\
\hline 0.10 & $1.191861879 \mathrm{E}+00$ & $1.2998 \mathrm{E}-06$ & $1.191860576 \mathrm{E}+00$ & $3.0532 \mathrm{E}-09$ & $1.191860580 \mathrm{E}+00$ \\
\hline 0.30 & $1.450247197 \mathrm{E}+00$ & $6.6333 \mathrm{E}-06$ & $1.450240549 \mathrm{E}+00$ & $1.5162 \mathrm{E}-08$ & $1.450240564 \mathrm{E}+00$ \\
\hline 0.40 & $1.443051695 \mathrm{E}+00$ & $1.1470 \mathrm{E}-05$ & $1.443040200 \mathrm{E}+00$ & $2.5913 \mathrm{E}-08$ & $1.443040226 \mathrm{E}+00$ \\
\hline 0.50 & $1.270398578 \mathrm{E}+00$ & $1.8532 \mathrm{E}-05$ & $1.270380005 \mathrm{E}+00$ & 4.1429E-08 & $1.270380046 \mathrm{E}+00$ \\
\hline 0.60 & $8.497854265 \mathrm{E}-01$ & $2.8660 \mathrm{E}-05$ & $8.497567028 \mathrm{E}-01$ & $6.3461 \mathrm{E}-08$ & $8.497567663 \mathrm{E}-01$ \\
\hline 0.80 & $-1.221576807 \mathrm{E}+00$ & $6.2986 \mathrm{E}-05$ & $-1.221639931 \mathrm{E}+00$ & $1.3714 \mathrm{E}-07$ & $-1.221639794 \mathrm{E}+00$ \\
\hline 0.90 & $-3.223453694 \mathrm{E}+00$ & $9.0665 \mathrm{E}-05$ & $-3.223544555 \mathrm{E}+00$ & $1.9595 \mathrm{E}-07$ & $-3.223544359 \mathrm{E}+00$ \\
\hline 1.00 & $-6.202224407 E+00$ & $1.2864 \mathrm{E}-04$ & $-6.202353319 E+00$ & $2.7612 \mathrm{E}-07$ & $-6.202353043 E+00$ \\
\hline 1.10 & $-1.050814302 \mathrm{E}+01$ & $1.8035 \mathrm{E}-04$ & $-1.050832375 \mathrm{E}+01$ & $3.8469 \mathrm{E}-07$ & $-1.050832337 \mathrm{E}+01$ \\
\hline 1.20 & $-1.660033426 \mathrm{E}+01$ & $2.5035 \mathrm{E}-04$ & $-1.660058514 \mathrm{E}+01$ & $5.3085 \mathrm{E}-07$ & $-1.660058461 \mathrm{E}+01$ \\
\hline 1.30 & $-2.507800780 \mathrm{E}+01$ & $3.4455 \mathrm{E}-04$ & $-2.507835308 \mathrm{E}+01$ & $7.2660 \mathrm{E}-07$ & $-2.507835236 \mathrm{E}+01$ \\
\hline 1.40 & $-3.672017589 E+01$ & 4.7072E-04 & $-3.672064759 E+01$ & $9.8758 \mathrm{E}-07$ & $-3.672064661 \mathrm{E}+01$ \\
\hline 1.50 & $-5.253611181 \mathrm{E}+01$ & $6.3893 \mathrm{E}-04$ & $-5.253675207 \mathrm{E}+01$ & $1.3341 \mathrm{E}-06$ & $-5.253675074 \mathrm{E}+01$ \\
\hline 1.70 & $-1.022788292 \mathrm{E}+02$ & $1.1579 \mathrm{E}-03$ & $-1.022799895 \mathrm{E}+02$ & $2.3966 \mathrm{E}-06$ & $-1.022799871 \mathrm{E}+02$ \\
\hline 1.80 & $-1.400413930 \mathrm{E}+02$ & $1.5476 \mathrm{E}-03$ & $-1.400429438 E+02$ & $3.1906 \mathrm{E}-06$ & $-1.400429407 \mathrm{E}+02$ \\
\hline 1.90 & $-1.898815579 \mathrm{E}+02$ & $2.0601 \mathrm{E}-03$ & $-1.898836222 \mathrm{E}+02$ & 4.2312E-06 & $-1.898836179 \mathrm{E}+02$ \\
\hline 2.00 & $-2.553351487 \mathrm{E}+02$ & $2.7320 \mathrm{E}-03$ & $-2.553378862 \mathrm{E}+02$ & 5.5912E-06 & $-2.553378806 \mathrm{E}+02$ \\
\hline 2.10 & $-3.409157287 \mathrm{E}+02$ & $3.6106 \mathrm{E}-03$ & $-3.409193466 \mathrm{E}+02$ & 7.3647E-06 & $-3.409193392 \mathrm{E}+02$ \\
\hline 2.20 & $-4.523746540 \mathrm{E}+02$ & 4.7569E-03 & $-4.523794205 E+02$ & $9.6720 \mathrm{E}-06$ & $-4.523794108 \mathrm{E}+02$ \\
\hline 2.30 & $-5.970283993 E+02$ & $6.2490 \mathrm{E}-03$ & $-5.970346610 \mathrm{E}+02$ & $1.2668 \mathrm{E}-05$ & $-5.970346483 \mathrm{E}+02$ \\
\hline 2.40 & $-7.841701997 \mathrm{E}+02$ & $8.1873 \mathrm{E}-03$ & $-7.841784036 \mathrm{E}+02$ & $1.6549 \mathrm{E}-05$ & $-7.841783870 \mathrm{E}+02$ \\
\hline 2.50 & $-1.025587304 \mathrm{E}+03$ & $1.0700 \mathrm{E}-02$ & $-1.025598026 \mathrm{E}+03$ & $2.1570 \mathrm{E}-05$ & $-1.025598004 \mathrm{E}+03$ \\
\hline 2.60 & $-1.336210431 \mathrm{E}+03$ & $1.3953 \mathrm{E}-02$ & $-1.336224411 \mathrm{E}+03$ & $2.8054 \mathrm{E}-05$ & $-1.336224383 \mathrm{E}+03$ \\
\hline 2.70 & $-1.734928626 \mathrm{E}+03$ & $1.8155 \mathrm{E}-02$ & $-1.734946817 \mathrm{E}+03$ & $3.6412 \mathrm{E}-05$ & $-1.734946780 \mathrm{E}+03$ \\
\hline 2.80 & $-2.245610919 \mathrm{E}+03$ & $2.3575 \mathrm{E}-02$ & $-2.245634540 \mathrm{E}+03$ & 4.7172E-05 & $-2.245634493 \mathrm{E}+03$ \\
\hline 2.90 & $-2.898386430 \mathrm{E}+03$ & $3.0556 \mathrm{E}-02$ & $-2.898417047 \mathrm{E}+03$ & $6.1003 \mathrm{E}-05$ & $-2.898416986 \mathrm{E}+03$ \\
\hline 3.00 & $-3.731247292 \mathrm{E}+03$ & $3.9534 \mathrm{E}-02$ & $-3.731286905 E+03$ & $7.8758 \mathrm{E}-05$ & $-3.731286826 \mathrm{E}+03$ \\
\hline
\end{tabular}

Table 3. Numerical approximations and maximum errors for step size $h=0.025$.

\begin{tabular}{|c|c|c|c|c|c|}
\hline \multirow{2}{*}{$t_{n}$} & \multicolumn{2}{|c|}{ RK4 Method $h=0.025$} & \multicolumn{2}{|c|}{ RK5 Method $h=0.025$} & \multirow{2}{*}{$\begin{array}{l}\text { Exact value } \\
z_{n}\end{array}$} \\
\hline & $z\left(t_{n}\right)$ & errors & $z\left(t_{n}\right)$ & errors & \\
\hline 0.00 & $1.000000000 \mathrm{E}+00$ & $0.0000 \mathrm{E}+00$ & $1.000000000 \mathrm{E}+00$ & $0.0000 \mathrm{E}+00$ & $1.000000000 \mathrm{E}+00$ \\
\hline 0.10 & $1.191860665 \mathrm{E}+00$ & 8.5920E-08 & $1.191860579 \mathrm{E}+00$ & $1.1046 \mathrm{E}-10$ & $1.191860580 \mathrm{E}+00$ \\
\hline 0.20 & $1.352109922 \mathrm{E}+00$ & $2.2438 \mathrm{E}-07$ & $1.352109697 \mathrm{E}+00$ & $2.8413 \mathrm{E}-10$ & $1.352109697 \mathrm{E}+00$ \\
\hline 0.30 & $1.450241002 \mathrm{E}+00$ & 4.3772E-07 & $1.450240563 \mathrm{E}+00$ & $5.4680 \mathrm{E}-10$ & $1.450240564 \mathrm{E}+00$ \\
\hline 0.40 & $1.443040982 \mathrm{E}+00$ & 7.5632E-07 & $1.443040225 \mathrm{E}+00$ & $9.3328 \mathrm{E}-10$ & $1.443040226 \mathrm{E}+00$ \\
\hline 0.50 & $1.270381267 \mathrm{E}+00$ & $1.2212 \mathrm{E}-06$ & $1.270380045 \mathrm{E}+00$ & $1.4903 \mathrm{E}-09$ & $1.270380046 \mathrm{E}+00$ \\
\hline 0.60 & $8.497586539 \mathrm{E}-01$ & $1.8876 \mathrm{E}-06$ & $8.497567640 \mathrm{E}-01$ & 2.2803E-09 & $8.497567663 \mathrm{E}-01$ \\
\hline 0.70 & 6.923920903E-02 & 2.8293E-06 & $6.923637637 \mathrm{E}-02$ & 3.3862E-09 & $6.923637976 \mathrm{E}-02$ \\
\hline 0.80 & $-1.221635649 \mathrm{E}+00$ & 4.1443E-06 & $-1.221639799 \mathrm{E}+00$ & 4.9180E-09 & $-1.221639794 \mathrm{E}+00$ \\
\hline 0.90 & $-3.223538396 \mathrm{E}+00$ & $5.9629 \mathrm{E}-06$ & $-3.223544366 \mathrm{E}+00$ & $7.0206 \mathrm{E}-09$ & $-3.223544359 \mathrm{E}+00$ \\
\hline 1.00 & $-6.202344586 \mathrm{E}+00$ & $8.4570 \mathrm{E}-06$ & $-6.202353053 \mathrm{E}+00$ & 9.8849E-09 & $-6.202353043 \mathrm{E}+00$ \\
\hline 1.10 & $-1.050831152 \mathrm{E}+01$ & $1.1853 \mathrm{E}-05$ & $-1.050832338 \mathrm{E}+01$ & $1.3761 \mathrm{E}-08$ & $-1.050832337 \mathrm{E}+01$ \\
\hline 1.20 & $-1.660056817 \mathrm{E}+01$ & $1.6447 \mathrm{E}-05$ & $-1.660058463 \mathrm{E}+01$ & $1.8976 \mathrm{E}-08$ & $-1.660058461 \mathrm{E}+01$ \\
\hline 1.30 & $-2.507832973 \mathrm{E}+01$ & $2.2629 \mathrm{E}-05$ & $-2.507835238 \mathrm{E}+01$ & $2.5956 \mathrm{E}-08$ & $-2.507835236 \mathrm{E}+01$ \\
\hline 1.40 & $-3.672061570 \mathrm{E}+01$ & $3.0906 \mathrm{E}-05$ & $-3.672064664 \mathrm{E}+01$ & $3.5256 \mathrm{E}-08$ & $-3.672064661 \mathrm{E}+01$ \\
\hline 1.50 & $-5.253670880 \mathrm{E}+01$ & 4.1939E-05 & $-5.253675078 \mathrm{E}+01$ & 4.7598E-08 & $-5.253675074 \mathrm{E}+01$ \\
\hline 1.60 & $-7.383016593 \mathrm{E}+01$ & $5.6587 \mathrm{E}-05$ & $-7.383022258 \mathrm{E}+01$ & $6.3914 \mathrm{E}-08$ & $-7.383022252 \mathrm{E}+01$ \\
\hline 1.70 & $-1.022799111 \mathrm{E}+02$ & $7.5965 \mathrm{E}-05$ & $-1.022799872 \mathrm{E}+02$ & $8.5414 \mathrm{E}-08$ & $-1.022799871 \mathrm{E}+02$ \\
\hline 1.80 & $-1.400428391 \mathrm{E}+02$ & $1.0151 \mathrm{E}-04$ & $-1.400429408 \mathrm{E}+02$ & $1.1366 \mathrm{E}-07$ & $-1.400429407 \mathrm{E}+02$ \\
\hline 1.90 & $-1.898834828 \mathrm{E}+02$ & $1.3510 \mathrm{E}-04$ & $-1.898836181 \mathrm{E}+02$ & $1.5065 \mathrm{E}-07$ & $-1.898836179 \mathrm{E}+02$ \\
\hline 2.00 & $-2.553377015 \mathrm{E}+02$ & $1.7913 \mathrm{E}-04$ & $-2.553378808 \mathrm{E}+02$ & $1.9899 \mathrm{E}-07$ & $-2.553378806 \mathrm{E}+02$ \\
\hline 2.10 & $-3.409191026 \mathrm{E}+02$ & 2.3669E-04 & $-3.409193395 \mathrm{E}+02$ & $2.6200 \mathrm{E}-07$ & $-3.409193392 \mathrm{E}+02$ \\
\hline 2.20 & $-4.523790990 \mathrm{E}+02$ & $3.1178 \mathrm{E}-04$ & $-4.523794112 \mathrm{E}+02$ & $3.4395 \mathrm{E}-07$ & $-4.523794108 \mathrm{E}+02$ \\
\hline 2.30 & $-5.970342388 \mathrm{E}+02$ & 4.0951E-04 & $-5.970346488 \mathrm{E}+02$ & 4.5030E-07 & $-5.970346483 \mathrm{E}+02$ \\
\hline 2.40 & $-7.841778506 \mathrm{E}+02$ & $5.3645 \mathrm{E}-04$ & $-7.841783876 \mathrm{E}+02$ & $5.8808 \mathrm{E}-07$ & $-7.841783870 \mathrm{E}+02$ \\
\hline 2.50 & $-1.025597303 \mathrm{E}+03$ & $7.0101 \mathrm{E}-04$ & $-1.025598005 \mathrm{E}+03$ & 7.6623E-07 & $-1.025598004 \mathrm{E}+03$ \\
\hline 2.60 & $-1.336223469 \mathrm{E}+03$ & $9.1396 \mathrm{E}-04$ & $-1.336224384 \mathrm{E}+03$ & $9.9620 \mathrm{E}-07$ & $-1.336224383 \mathrm{E}+03$ \\
\hline 2.70 & $-1.734945591 \mathrm{E}+03$ & $1.1890 \mathrm{E}-03$ & $-1.734946781 \mathrm{E}+03$ & $1.2926 \mathrm{E}-06$ & $-1.734946780 \mathrm{E}+03$ \\
\hline 2.80 & $-2.245632949 \mathrm{E}+03$ & $1.5438 \mathrm{E}-03$ & $-2.245634495 \mathrm{E}+03$ & $1.6741 \mathrm{E}-06$ & $-2.245634493 \mathrm{E}+03$ \\
\hline 2.90 & $-2.898414985 \mathrm{E}+03$ & $2.0008 \mathrm{E}-03$ & $-2.898416988 \mathrm{E}+03$ & $2.1643 \mathrm{E}-06$ & $-2.898416986 \mathrm{E}+03$ \\
\hline 3.00 & $-3.731284238 \mathrm{E}+03$ & $2.5884 \mathrm{E}-03$ & $-3.731286829 \mathrm{E}+03$ & $2.7934 \mathrm{E}-06$ & $-3.731286826 \mathrm{E}+03$ \\
\hline
\end{tabular}


Table 4. Numerical approximations and maximum errors for step size $h=0.0125$.

\begin{tabular}{|c|c|c|c|c|c|}
\hline \multirow{2}{*}{$t_{n}$} & \multicolumn{2}{|c|}{ RK4 Method h=0.0125 } & \multicolumn{2}{|c|}{ RK5 Method h=0.0125 } & \multirow{2}{*}{$\begin{array}{l}\text { Exact value } \\
z_{n}\end{array}$} \\
\hline & $z\left(t_{n}\right)$ & errors & $z\left(t_{n}\right)$ & errors & \\
\hline 0.00 & $1.000000000 \mathrm{E}+00$ & $0.0000 \mathrm{E}+00$ & $1.000000000 \mathrm{E}+00$ & $0.0000 \mathrm{E}+00$ & $1.000000000 \mathrm{E}+00$ \\
\hline 0.10 & $1.191860585 \mathrm{E}+00$ & 5.5223E-09 & $1.191860579 \mathrm{E}+00$ & $3.7019 \mathrm{E}-12$ & $1.191860580 \mathrm{E}+00$ \\
\hline 0.20 & $1.352109712 \mathrm{E}+00$ & $1.4415 \mathrm{E}-08$ & $1.352109697 \mathrm{E}+00$ & $9.5168 \mathrm{E}-12$ & $1.352109697 \mathrm{E}+00$ \\
\hline 0.30 & $1.450240592 \mathrm{E}+00$ & $2.8110 \mathrm{E}-08$ & $1.450240564 \mathrm{E}+00$ & $1.8304 \mathrm{E}-11$ & $1.450240564 \mathrm{E}+00$ \\
\hline 0.40 & $1.443040274 \mathrm{E}+00$ & 4.8553E-08 & $1.443040226 \mathrm{E}+00$ & $3.1220 \mathrm{E}-11$ & $1.443040226 \mathrm{E}+00$ \\
\hline 0.50 & $1.270380124 \mathrm{E}+00$ & $7.8375 \mathrm{E}-08$ & $1.270380046 \mathrm{E}+00$ & $4.9825 \mathrm{E}-11$ & $1.270380046 \mathrm{E}+00$ \\
\hline 0.60 & $8.497568874 \mathrm{E}-01$ & $1.2111 \mathrm{E}-07$ & $8.497567662 \mathrm{E}-01$ & $7.6200 \mathrm{E}-11$ & 8.497567663E-01 \\
\hline 0.70 & $6.923656123 \mathrm{E}-02$ & $1.8148 \mathrm{E}-07$ & $6.923637965 \mathrm{E}-02$ & $1.1311 \mathrm{E}-10$ & $6.923637976 \mathrm{E}-02$ \\
\hline 0.80 & $-1.221639528 \mathrm{E}+00$ & $2.6576 \mathrm{E}-07$ & $-1.221639794 \mathrm{E}+00$ & $1.6421 \mathrm{E}-10$ & $-1.221639794 \mathrm{E}+00$ \\
\hline 0.90 & $-3.223543977 \mathrm{E}+00$ & $3.8230 \mathrm{E}-07$ & $-3.223544359 \mathrm{E}+00$ & $2.3434 \mathrm{E}-10$ & $-3.223544359 \mathrm{E}+00$ \\
\hline 1.00 & $-6.202352501 E+00$ & $5.4210 \mathrm{E}-07$ & $-6.202353044 \mathrm{E}+00$ & $3.2983 \mathrm{E}-10$ & $-6.202353043 \mathrm{E}+00$ \\
\hline 1.10 & $-1.050832261 \mathrm{E}+01$ & 7.5963E-07 & $-1.050832337 \mathrm{E}+01$ & $4.5902 \mathrm{E}-10$ & $-1.050832337 \mathrm{E}+01$ \\
\hline 1.20 & $-1.660058356 \mathrm{E}+01$ & $1.0539 \mathrm{E}-06$ & $-1.660058461 \mathrm{E}+01$ & $6.3280 \mathrm{E}-10$ & $-1.660058461 \mathrm{E}+01$ \\
\hline 1.30 & $-2.507835091 \mathrm{E}+01$ & $1.4498 \mathrm{E}-06$ & $-2.507835236 \mathrm{E}+01$ & $8.6533 \mathrm{E}-10$ & $-2.507835236 \mathrm{E}+01$ \\
\hline 1.40 & $-3.672064463 \mathrm{E}+01$ & $1.9798 \mathrm{E}-06$ & $-3.672064661 \mathrm{E}+01$ & $1.1751 \mathrm{E}-09$ & $-3.672064661 \mathrm{E}+01$ \\
\hline 1.50 & $-5.253674805 \mathrm{E}+01$ & $2.6862 \mathrm{E}-06$ & $-5.253675074 \mathrm{E}+01$ & $1.5861 \mathrm{E}-09$ & $-5.253675074 \mathrm{E}+01$ \\
\hline 1.60 & $-7.383021889 \mathrm{E}+01$ & $3.6240 \mathrm{E}-06$ & $-7.383022252 \mathrm{E}+01$ & 2.1294E-09 & $-7.383022252 \mathrm{E}+01$ \\
\hline 1.70 & $-1.022799822 \mathrm{E}+02$ & 4.8644E-06 & $-1.022799871 \mathrm{E}+02$ & $2.8451 \mathrm{E}-09$ & $-1.022799871 \mathrm{E}+02$ \\
\hline 1.80 & $-1.400429342 \mathrm{E}+02$ & $6.4998 \mathrm{E}-06$ & $-1.400429407 \mathrm{E}+02$ & 3.7851E-09 & $-1.400429407 \mathrm{E}+02$ \\
\hline 1.90 & $-1.898836093 \mathrm{E}+02$ & 8.6494E-06 & $-1.898836179 \mathrm{E}+02$ & 5.0163E-09 & $-1.898836179 \mathrm{E}+02$ \\
\hline 2.00 & $-2.553378692 \mathrm{E}+02$ & $1.1467 \mathrm{E}-05$ & $-2.553378806 \mathrm{E}+02$ & $6.6247 \mathrm{E}-09$ & $-2.553378806 \mathrm{E}+02$ \\
\hline 2.10 & $-3.409193241 \mathrm{E}+02$ & $1.5151 \mathrm{E}-05$ & $-3.409193393 \mathrm{E}+02$ & 8.7195E-09 & $-3.409193392 \mathrm{E}+02$ \\
\hline 2.20 & $-4.523793909 E+02$ & $1.9955 \mathrm{E}-05$ & $-4.523794108 \mathrm{E}+02$ & $1.1443 \mathrm{E}-08$ & $-4.523794108 \mathrm{E}+02$ \\
\hline 2.30 & $-5.970346221 \mathrm{E}+02$ & 2.6209E-05 & $-5.970346483 E+02$ & $1.4976 \mathrm{E}-08$ & $-5.970346483 \mathrm{E}+02$ \\
\hline 2.40 & $-7.841783527 \mathrm{E}+02$ & $3.4330 \mathrm{E}-05$ & $-7.841783870 \mathrm{E}+02$ & $1.9552 \mathrm{E}-08$ & $-7.841783870 \mathrm{E}+02$ \\
\hline 2.50 & $-1.025597959 \mathrm{E}+03$ & $4.4858 \mathrm{E}-05$ & $-1.025598004 \mathrm{E}+03$ & $2.5466 \mathrm{E}-08$ & $-1.025598004 \mathrm{E}+03$ \\
\hline 2.60 & $-1.336224325 \mathrm{E}+03$ & $5.8480 \mathrm{E}-05$ & $-1.336224383 \mathrm{E}+03$ & $3.3100 \mathrm{E}-08$ & $-1.336224383 \mathrm{E}+03$ \\
\hline 2.70 & $-1.734946704 \mathrm{E}+03$ & $7.6076 \mathrm{E}-05$ & $-1.734946780 \mathrm{E}+03$ & 4.2936E-08 & $-1.734946780 \mathrm{E}+03$ \\
\hline 2.80 & $-2.245634394 \mathrm{E}+03$ & $9.8770 \mathrm{E}-05$ & $-2.245634493 \mathrm{E}+03$ & 5.5591E-08 & $-2.245634493 \mathrm{E}+03$ \\
\hline 2.90 & $-2.898416858 \mathrm{E}+03$ & $1.2799 \mathrm{E}-04$ & $-2.898416986 \mathrm{E}+03$ & 7.1851E-08 & $-2.898416986 \mathrm{E}+03$ \\
\hline 3.00 & $-3.731286660 \mathrm{E}+03$ & $1.6558 \mathrm{E}-04$ & $-3.731286826 \mathrm{E}+03$ & $9.2713 \mathrm{E}-08$ & $-3.731286826 \mathrm{E}+03$ \\
\hline
\end{tabular}

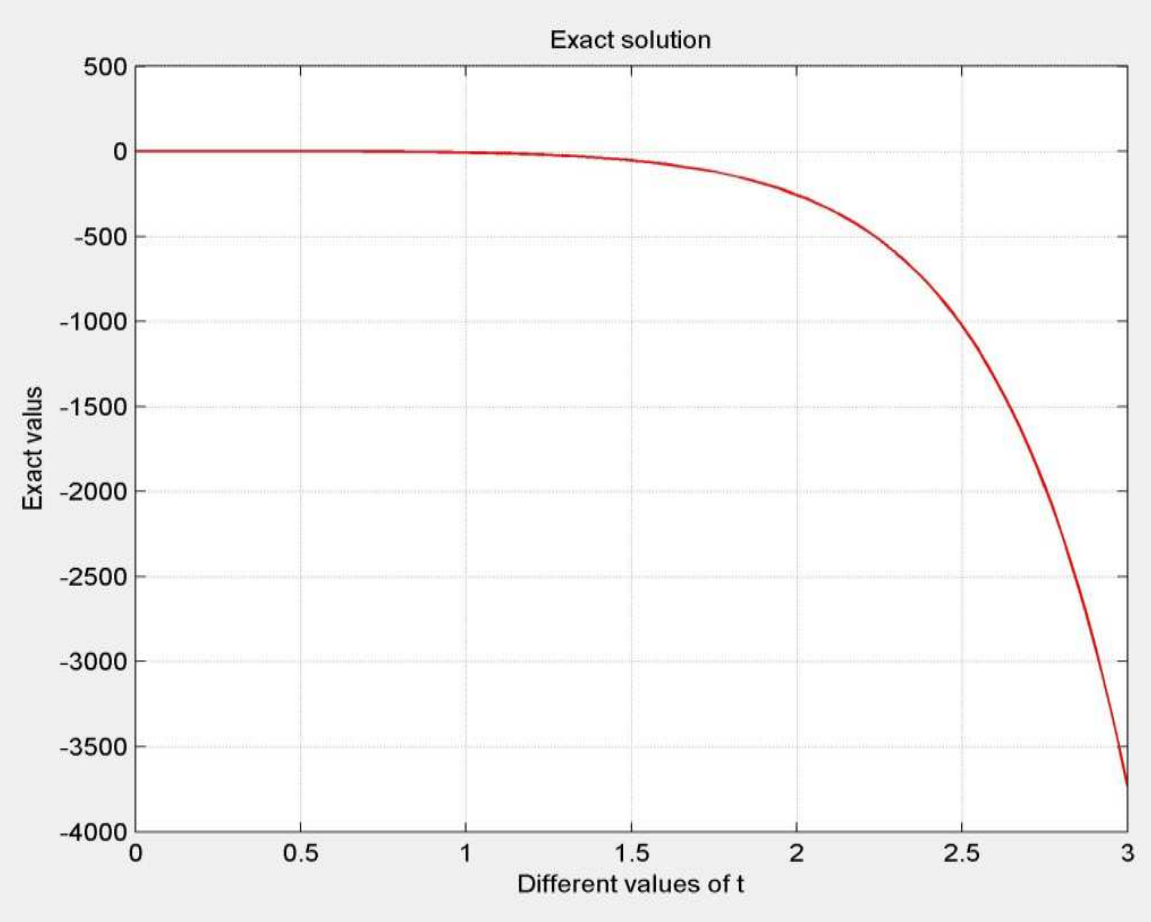

Figure 1. Exact numerical solutions. 


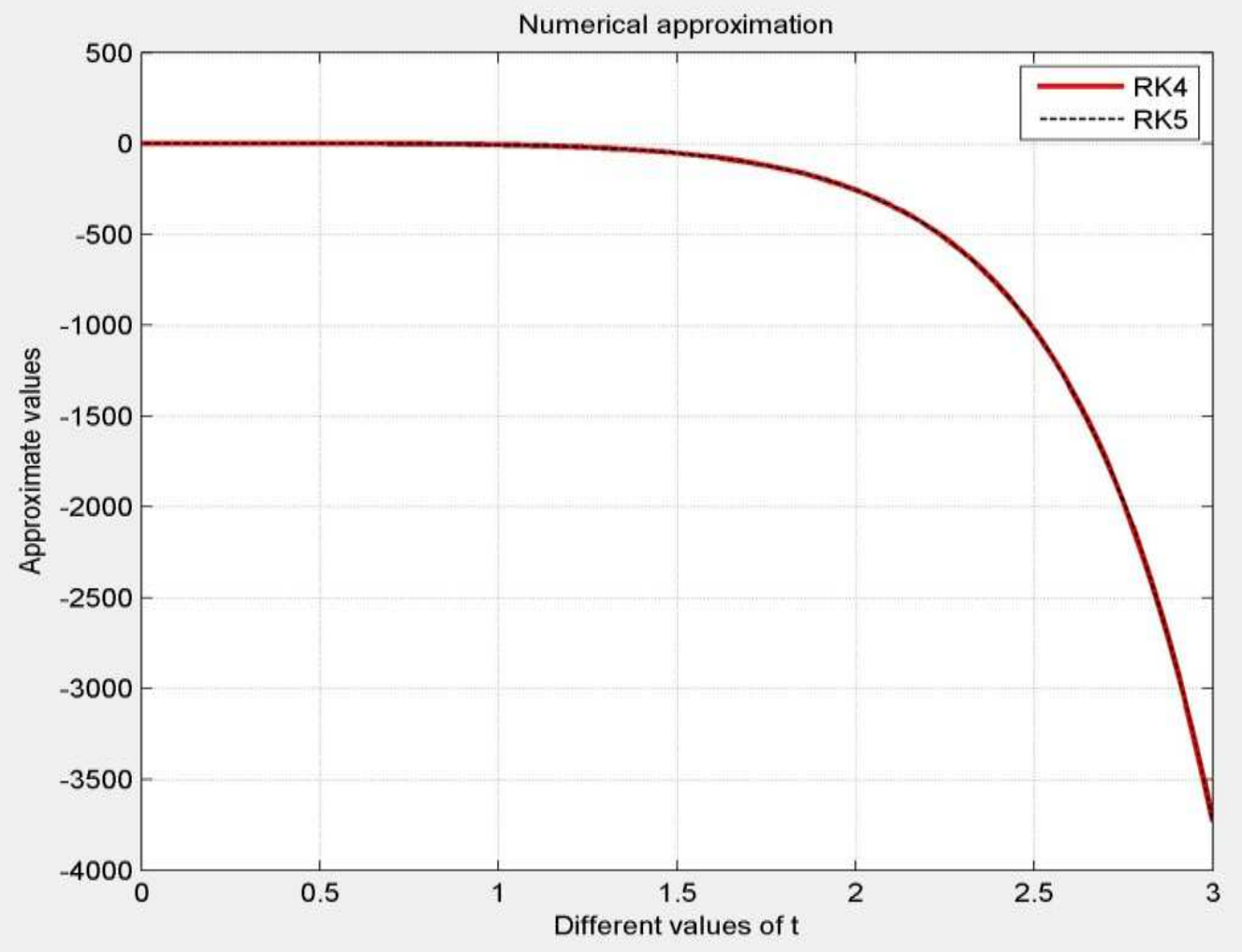

Figure 2. Approximate numerical solutions.

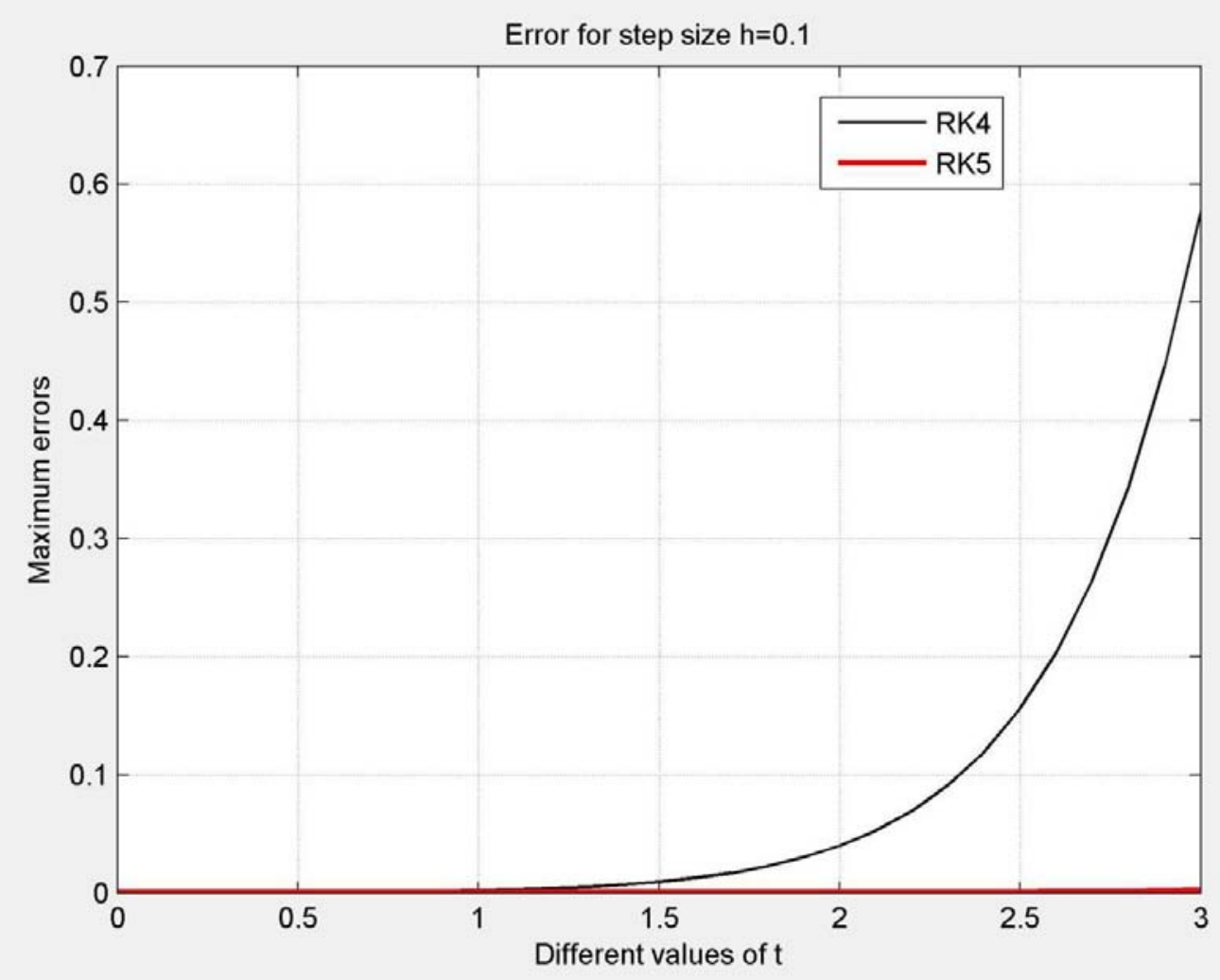

Figure 3. Error for step size $h=0.1$. 


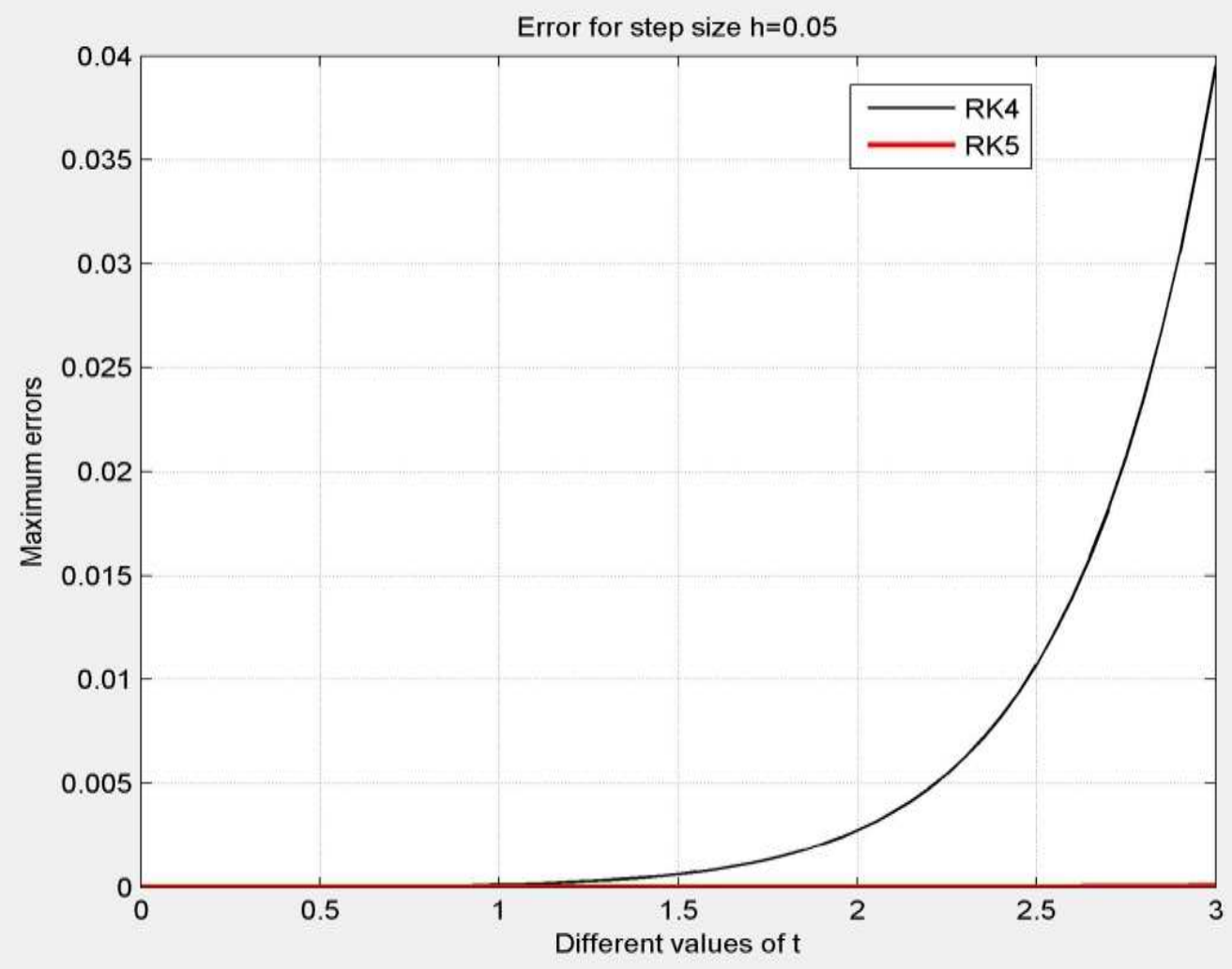

Figure 4. Error for step size $h=0.05$.

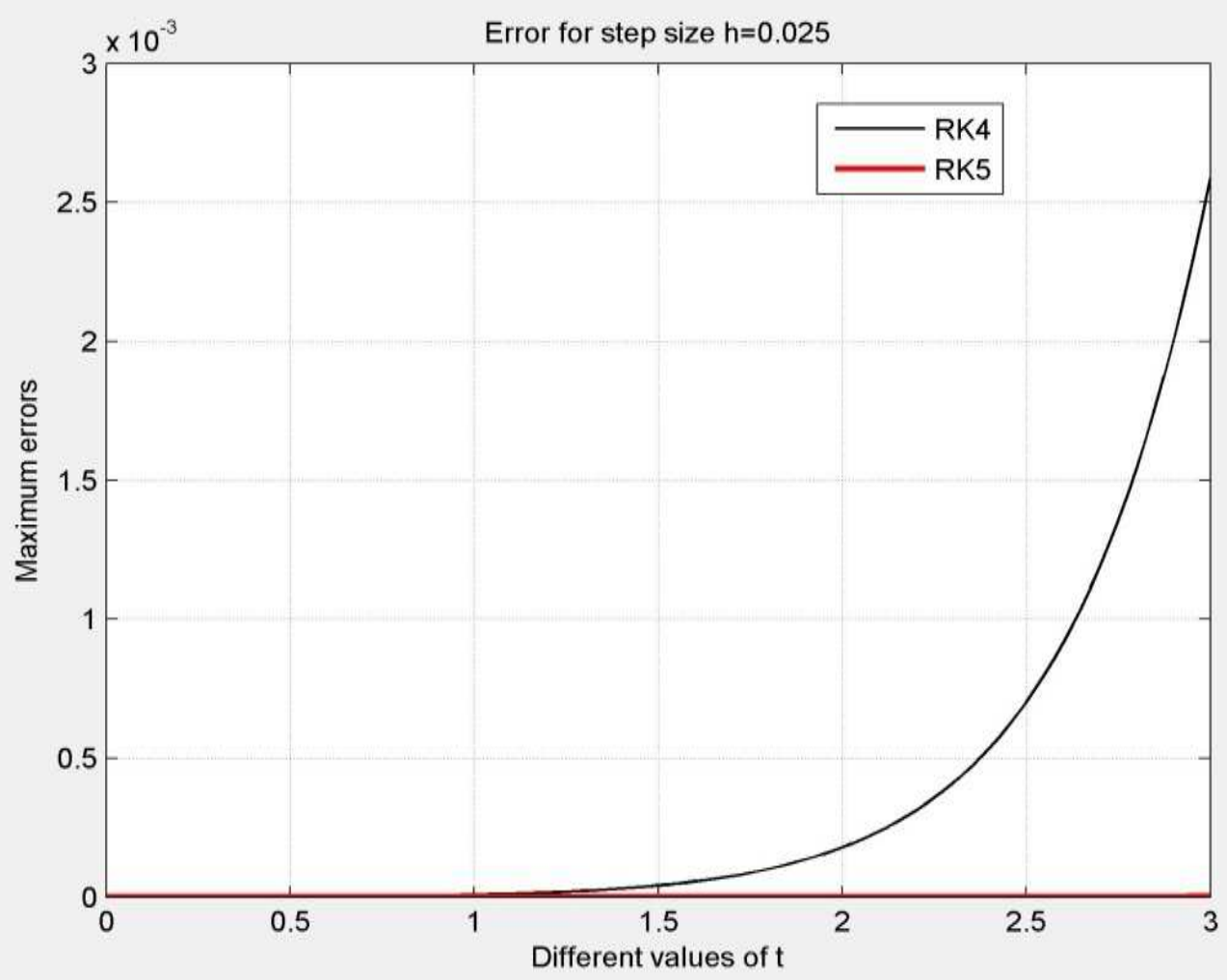

Figure 5. Error for step size $h=0.025$. 


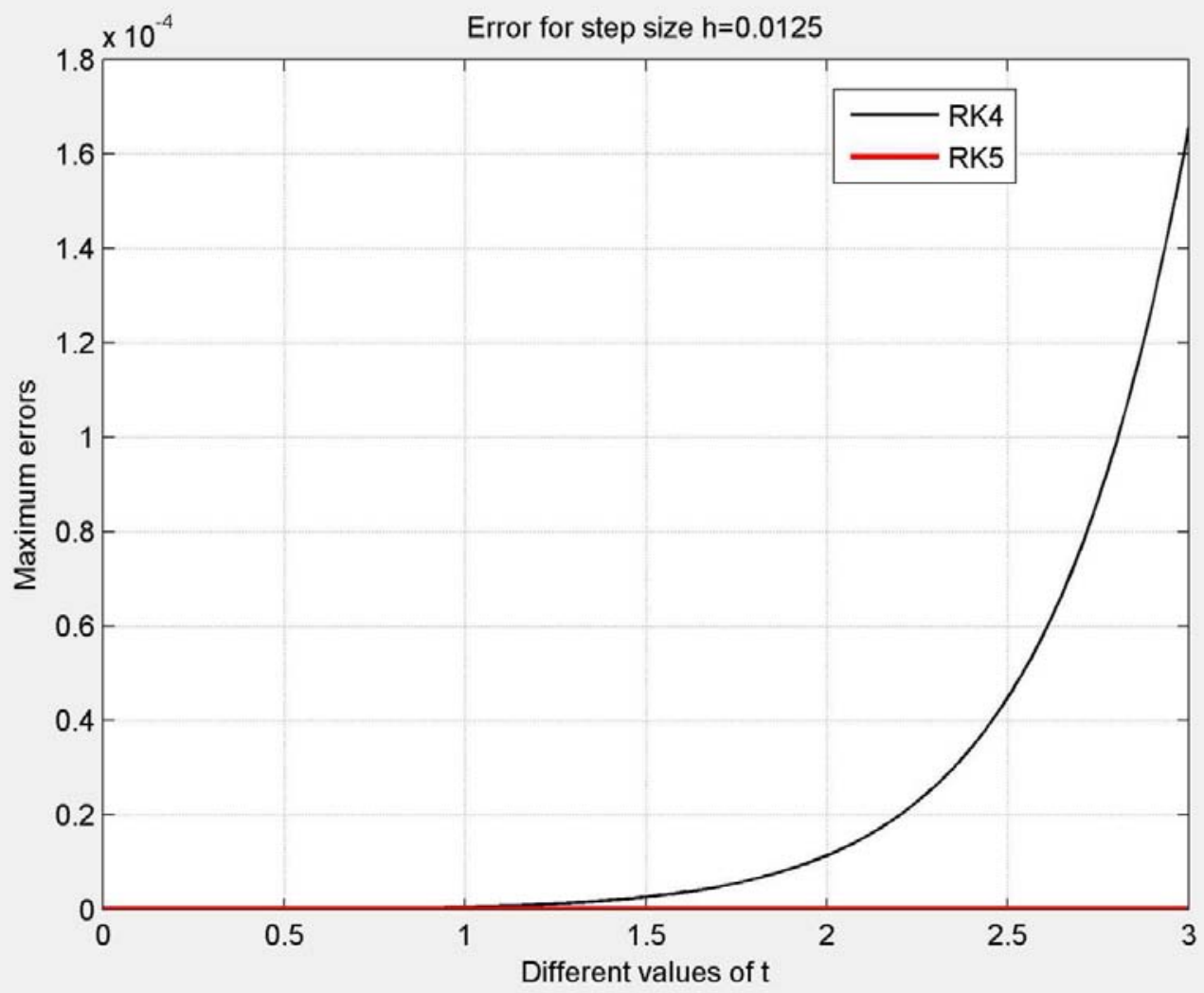

Figure 6. Error for step size $h=0.0125$.

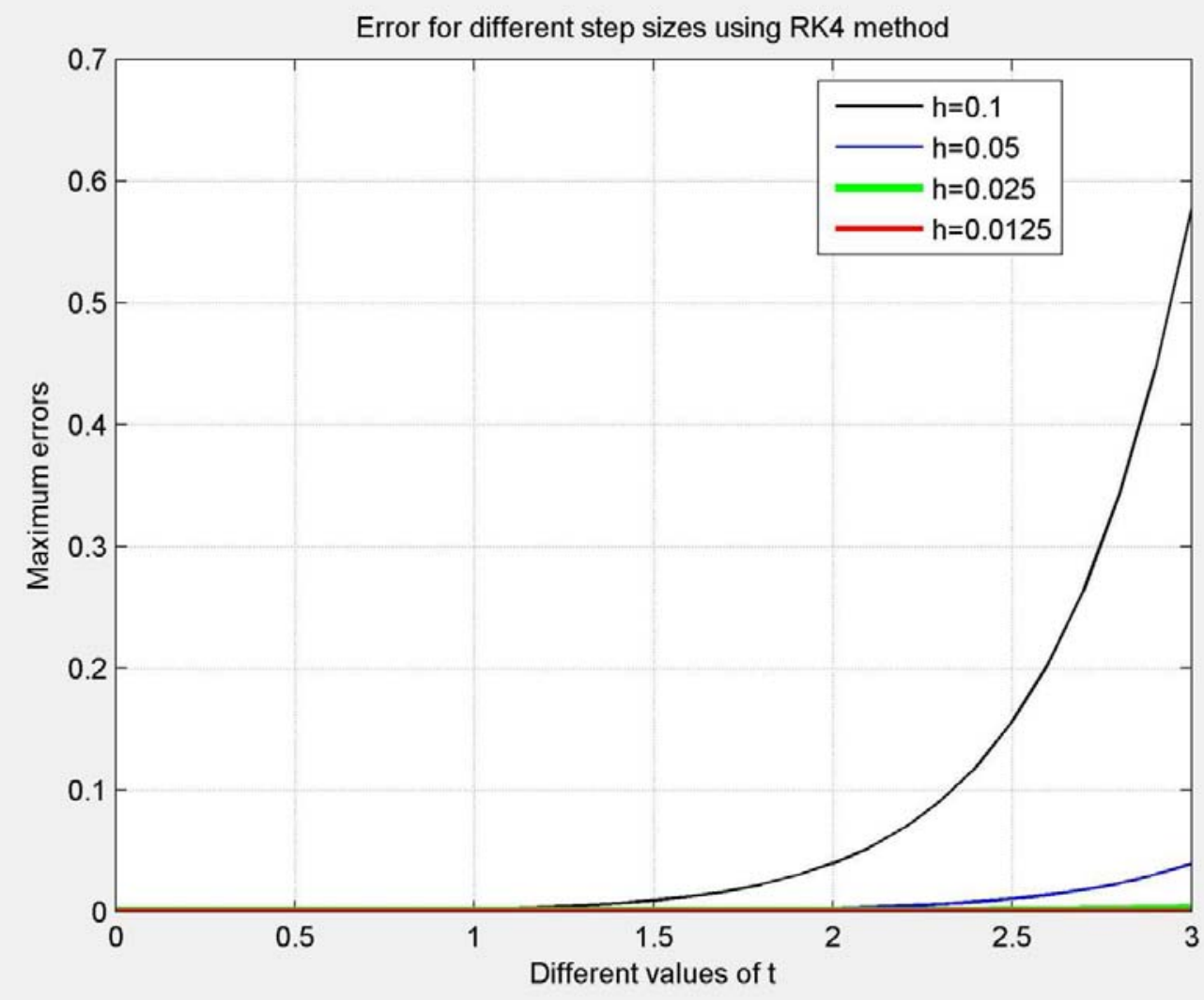

Figure 7. Error for different step sizes using RK4 method. 


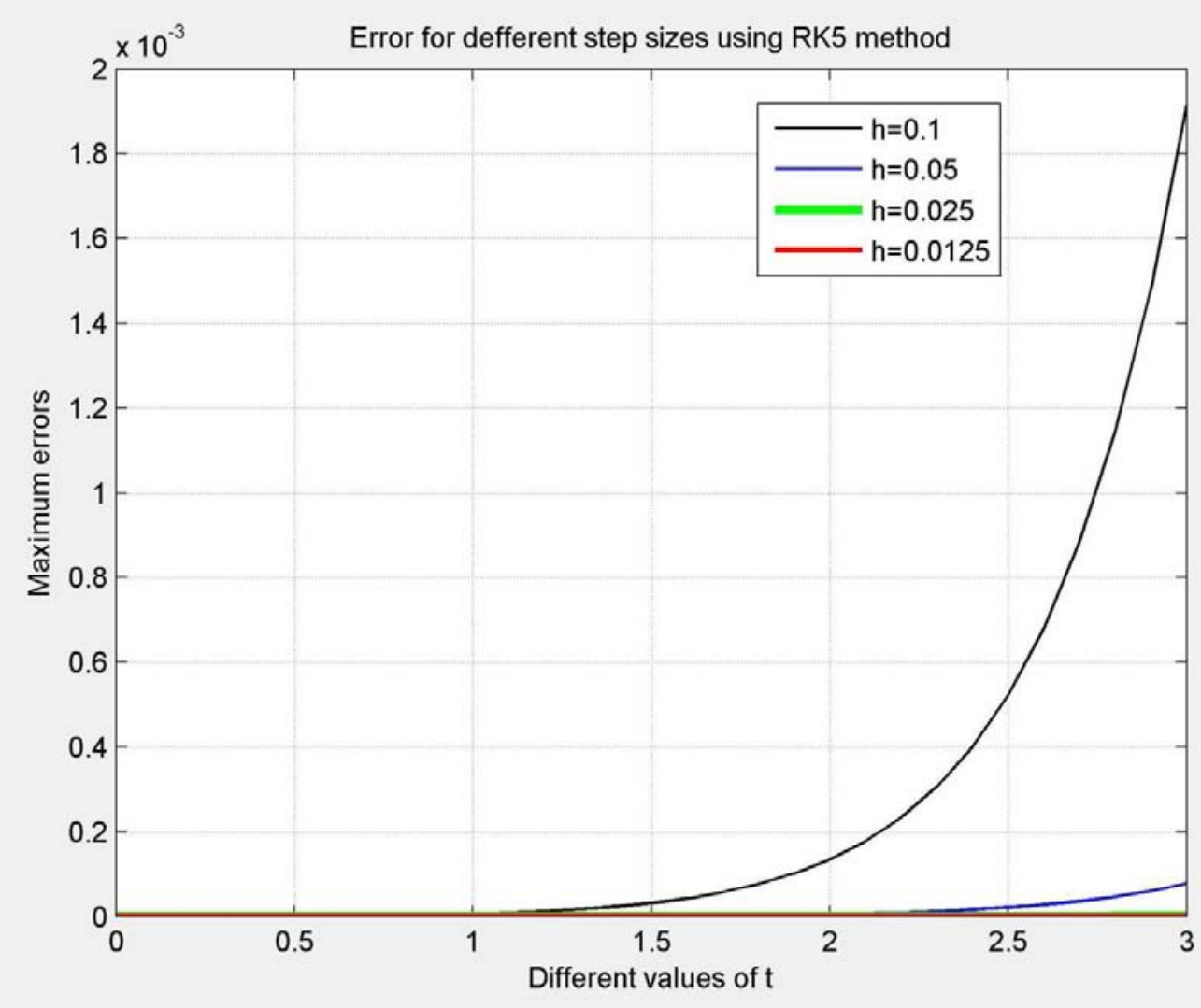

Figure 8. Error for different step sizes using RK5 method.

\section{Discussion}

The acquired results are displayed in Tables 1-4 and graphically presented in Figures (1-8). The approximate solutions and maximum errors are calculated with the step sizes $0.1,0.05,0.025$ and 0.0125 and also compared to the exact solutions. Also from Tables 1-4 we observe that RK5 method gives more accurate results and better than RK4 method. This argument is also cleared by Figures 3-6. From all Tables and Figures for each method we say that a numerical solution converges to the exact solution if the step size is decreased. Also from figures 7 and 8 we conclude that if the step size tends to zero then the errors also tends to zero.

\section{Conclusion}

In this paper, fourth order Runge-Kutta method and Butcher's fifth order Runge-Kutta method are applied to solve third order initial value problem (IVP) of ordinary differential equation (ODE). To find more accurate results we reduced the step size for both the methods. From the resulting tables and figure we have analyzed that the solutions of both methods are converges to the exact solutions for decreasing the step size $h$. From the figure 2 we see that both methods give almost same results but from figures 3-6 it is clear that RK5 method gives more accurate results than RK4 method. We state that the Butcher's fifth order Runge-Kutta method is more appropriate and proficient for finding the numerical solutions of initial value problems (IVP) than fourth order Runge-Kutta method. Hence from this study it is clear that to find more accurate results higher order methods are appropriate than lower order methods.

\section{References}

[1] Rabiei, F., \& Ismail, F. (2012). Fifth-order Improved RungeKutta method for solving ordinary differential equation. Australian Journal of Basic and Applied Sciences, 6 (3), 97-105.

[2] Butcher, J. C. (1995). On fifth order Runge-Kutta methods. BIT Numerical Mathematics, 35 (2), 202-209.

[3] Islam, M. A. (2015). A Comparative Study on Numerical Solutions of Initial Value Problems (IVP) for Ordinary Differential Equations (ODE) with Euler and Runge-kutta Methods. American Journal of Computational Mathematics, 5 (03), 393.

[4] Butcher, J. C. (1964). On Runge-Kutta processes of high order. Journal of the Australian Mathematical Society, 4 (02), 179-194.

[5] Butcher, J. C. (1996). A history of Runge-Kutta methods. Applied numerical mathematics, 20 (3), 247-260.

[6] Islam, M. A. (2015). Accuracy Analysis of Numerical solutions of Initial Value Problems (IVP) for Ordinary Differential Equations (ODE). IOSR Journal of Mathematics, $11,18-23$. 
[7] Goeken, D., \& Johnson, O. (2000). Runge-Kutta with higher order derivative approximations. Applied numerical mathematics, 34 (2-3), 207-218.

[8] Lambert, J. D. (1973). Computational methods in ordinary differential equations. Wiley, New York.

[9] Hall, G. and Watt, J. M. (1976) Modern Numerical Methods for Ordinary Differential Equations. Oxford University Press, Oxford.

[10] Mathews, J. H. (2005) Numerical Methods for Mathematics, Science and Engineering. Prentice-Hall, India.
[11] Gerald, C. F. and Wheatley, P. O. (2002) Applied Numerical Analysis. Pearson Education, India.

[12] Burden, R. L. and Faires, J. D. (2002) Numerical Analysis. Bangalore, India.

[13] Sastry, S. S. (2000) Introductory Methods of Numerical Analysis. Prentice-Hall, India.

[14] Balagurusamy, E. (2006) Numerical Methods. Tata McGrawHill, New Delhi.

[15] Hossain, Md. S., Bhattacharjee, P. K. and Hossain, Md. E. (2013) Numerical Analysis. Titas Publications, Dhaka. 